\title{
CARACTERIZAÇÃO FLORÍSTICA, FITOSSOCIOLÓGICA E REGENERAÇÃO NATURAL DO SUB-BOSQUE DA RESERVA GENÉTICA FLORESTAL TAMANDUÁ, DF
}

\section{PHYTOSOCIOGICAL AND FLORISTIC CHARACTERIZATION AND NATURAL REGENERATION OF THE UNDERGROWTH AT TAMANDUA FOREST GENETIC RESERVE, BRAZIL.}

\author{
José Alves da Silva ${ }^{1}$ Edson Junqueira Leite ${ }^{2}$ Marcio Silveira ${ }^{3}$ \\ Armando Antonieta Nassif ${ }^{4}$ Salomão João Marcelo de Rezende ${ }^{5}$
}

\section{RESUMO}

O presente trabalho, desenvolvido na Reserva Genética Florestal Tamanduá, DF, teve como objetivo efetuar o levantamento fitossociológico, composição florística e distribuição espacial dos indivíduos das espécies arbóreas ocorrentes no sub-bosque da Reserva, focalizando Cariniana estrellensis (Raddi) O. Kuntze, Apuleia leiocarpa (Vogel) J. F.Macbr., Copaifera langsdorffii Desf., Anadenanthera macrocarpa (Benth.) Brenan, Virola sebifera Aubl., Hymenaea courbaril var. stilbocarpa (Hayne) Lee \& Lang., Aspidosperma discolor A. St. Hil., Astronium fraxinifolium Schott e Schefflera morototoni B. Maguire, Stey. \& Frodin, consideradas prioritárias para conservação in situ. Foram utilizados cinco transectos com $10 \mathrm{~m}$ de largura cada, alocados eqüidistantes e de comprimento variável, demarcados perpendicularmente ao curso da drenagem principal. Foram encontrados 21.482 regenerantes ha ${ }^{-1}$ em 69 espécies vegetais, 53 gêneros e 39 famílias botânicas. Em número de espécies, as famílias que mais se destacaram entre os regenerantes foram, em ordem decrescente, Leguminosae, Rubiaceae, Sapindaceae e Meliaceae. Entre os jovens, as famílias com maiores valor de importância (VI) foram pela ordem Meliaceae (32,78 \%), Rubiceae (13,92 \%), Burseraceae $(13,76 \%)$, Rutaceae $(8,54 \%)$ e Hippocrateaceae $(6,36)$, totalizando $75,36 \%$ de valor de importância e 78,56 $\%$ de valor de cobertura. Todas as espécies objeto deste trabalho ocorreram entre os regenerantes, destacandose Cariniana estrellensis, porém, apenas Copaifera lagsdorffii e Virola sebifera ocorreram entre os indivíduos jovens. O quociente de mistura de 1:3 indicou tratar-se de uma mata rica em espécies, comparativamente a outros tipos florestais. A distribuição espacial dos regenerantes das espécies em questão mostrou padrões diferenciados.

Palavras-chave: fitossociologia; composição florística; regeneração natural; mata de galeria.

\begin{abstract}
This work was carried out in the Tamanduá Forest Genetic Reserve, in Brasília, Brazil, comprised mainly by gallery forest. It aimed to assess the floristic composition, natural regeneration and the spatial distribution of the main woody species in the undergrowth. The species were Cariniana estrellensis (Raddi) O. Kuntze, Apuleia leiocarpa (Vogel) J. F.Macbr., Copaifera langsdorffii Desf., Anadenanthera macrocarpa (Benth.) Brenan, Virola sebifera Aubl., Hymenaea courbaril var. stilbocarpa (Hayne) Lee \& Lang., Aspidosperma discolor A. St. Hil., Astronium fraxinifolium Schott and Schefflera morototoni B. Maguire, Stey. \& Frodin, all considered priorities for in situ conservation. Five transects were used, each 10 meters wide, laid perpendicular to the main watercourse in the studied area. A total of 21,482 regenerants per hectare were found in 69 species, 53 genera and 39 families. The families which had the largest numbers of species as seedlings were, in descending order, Leguminosae (Fabaceae), Rubiaceae, Sapindaceae and Meliaceae. Surveying of young individuals, the dominant families with higher importance value (IV) were as follows: Meliaceae (32.78 \%), Rubiaceae (13.92\%), Burseraceae (13.76 \%), Rutaceae (8.54 \%) and Hippocrateaceae
\end{abstract}

1. Engenheiro Florestal, Dr., Pesquisador da Embrapa Recursos Genéticos e Biotecnologia, CEP 70770.900, Brasília (DF). jalves@ cenargen.embrapa.br

2. Engenheiro Florestal, PhD., Pesquisador da Embrapa Recursos Genéticos e Biotecnologia, CEP 70770.900, Brasília (DF). edson@cenargen.emrapa.br

3. Biólogo, MSc., Embrapa Sede, CEP 70770.901, Brasília (DF). marcio.armando@embrapa.br

4. Engenheiro Florestal, MSc., Pesquisador Embrapa Recursos Genéticos e Biotecnologia, CEP 70770.900, Brasília (DF). antoniet@cenargen.embrapa.br

5. Engenheiro Florestal, Embrapa Recursos Genéticos e Biotecnologia, CEP 70770.900, Brasília (DF). genipapo@bol.com.br

Recebido para publicação em 31/03/2003 e aceito em 12/04/2004. 
(6.36\%), accounting for $75.36 \%$ of total IV and $78.56 \%$ of cover. All target species studied were represented among regenerants, especially Cariniana estrelllensis. However, only Copaifera langsdorffii and Virola sebifera were found among young individuals. A mixture quotient of 1:3 was calculated and indicates a forest rich in species, when compared to other similar forest formations. The spatial distribution of the regenerant species showed different patterns.

Key words: phytosociology; floristic composition; natural regeneration; gallery forest.

\section{INTRODUÇÃO}

As matas de galeria se distribuem como uma extensa malha por toda a região do Cerrado, cobrindo cerca de 5\% da extensão total da região. (Fonseca, 1992).

Essas matas são catalizadoras e mantenedoras da diversidade de espécies, desempenhando importante papel como refúgio natural da abundante fauna do Cerrado durante a estação seca, quando o abrigo, umidade e alimento são escassos nas formações savânicas e campestres. Funcionam como corredores entre reservas (Dias, 1992), contribuindo para aumentar o seu tamanho efetivo por favorecer o fluxo gênico entre indivíduos da flora e a dispersão de espécies florestais.(Paula et al., 1990; Schiavini, 1992; Martins Netto,1992).

O caráter de isolamento ou de fragmentação desse tipo florestal tem contribuído para colocar em risco a sobrevivência de determinadas espécies, onde a quebra de interações ecológicas pode levar à instabilidade, resultando no desaparecimento de genótipos, comprometendo as futuras descendências.(Kageyama e PatiñoValera, 1985; Roche, 1987).

À semelhança das florestas tropicais úmidas de interflúvio, as matas de galeria contêm grande número de espécies vegetais por unidade de área, porém sem predominância de nenhuma (Kageyama e Patiño-Valera, 1985; Roche, 1987). O distúrbio no dossel (clareiras) em florestas tropicais e mesmo em matas de galeria, torna-se muito importante na sua dinâmica de crescimento, pois o aumento da disponibilidade do recurso luz favorece o surgimento das espécies pioneiras (intolerantes), colonizadoras de clareiras no estágio inicial de sucessão, onde geralmente predominam (Whitmore, 1996). Essas espécies tendem a perder sua posição dominante para as espécies mais tolerantes, em razão das mudanças nas condições ambientais locais, em razão ao fechamento do dossel que impede o recrutamento de novos indivíduos intolerantes (Mory e Jardim, 2001). Entretanto, espécies que se caracterizam pelo contínuo recrutamento (curta viabilidade das sementes) apresentam alta mortalidade nos menores indivíduos, com menos chances competitivas, justificando, assim, sua forma de distribuição diamétrica exponencial (J invertido) (Mory e Jardim, 2001).

Com relação à estrutura, deve-se salientar que a permanência de determinada espécie em uma comunidade natural depende, não só da ocorrência de uma distribuição exponencial do número de indivíduos por classes de diâmetro, de tal modo que possa garantir sua estabilidade futura, mas também da existência de árvores reprodutivas. Desse modo, baixas densidades populacionais de adultos e regenerantes são indicativos de que a espécie poderá vir a ser substituída durante a evolução da floresta.

O objetivo deste trabalho foi analisar a estrutura fitossociológica, a diversidade florística e a distribuição espacial dos regenerantes relativos às espécies arbóreas seguintes, consideradas prioritárias para conservação in situ na Reserva Genética Florestal Tamanduá: Cariniana estrellensis (Raddi) O. Kuntze., Apuleia leiocarpa (Vogel) J. F. Macbr., Copaifera langsdorffii Desf., Anadenanthera macrocarpa (Benth.) Brenan, Virola sebifera Aubl., Hymenaea courbaril var. stilbocarpa (Hayne) Lee \& Lang., Aspidosperma discolor A. St. Hil., Astronium fraxinifolium Schott e Schefflera morototoni B. Maguire, Stey. \& Frondin.

\section{MATERIAL E MÉTODOS}

\section{Área de estudo}

A Reserva Genética Florestal Tamanduá é constituída por uma mata de galeria semidecídua, com solos distróficos (Eiten,1990), onde, entretanto, se pôde constatar a ocorrência de espécies indicadoras de terrenos rochosos de origem calcária (Astroniun fraxinifolium Schott), conforme relatado por Martins Netto (1992) e Silva et al., (2002). A mata possui uma área de 21,08 ha, com forma irregular que acompanha e 
protege as margens do riacho Tamanduá, porém, sem encharcamento ou inundação. As coordenadas geográficas de $15^{\circ} 56^{\prime} 00^{\prime \prime}$ de latitude Sul e 48 $10^{\circ} 00^{\prime \prime}$ de longitude Oeste, definem a posição central da Reserva que se situa nas proximidades da cidade satélite do Gama-DF, a uma altitude média de 997 metros (Figura 1). O clima predominante (Köppen) é de savana (Aw), com temperatura média anual de $28^{\circ} \mathrm{C}$ e média mínima de $15,9^{\circ} \mathrm{C}$, umidade relativa média anual de $60 \%$ e precipitação média anual de $1291 \mathrm{~mm}$ (Ayoade, 1983).

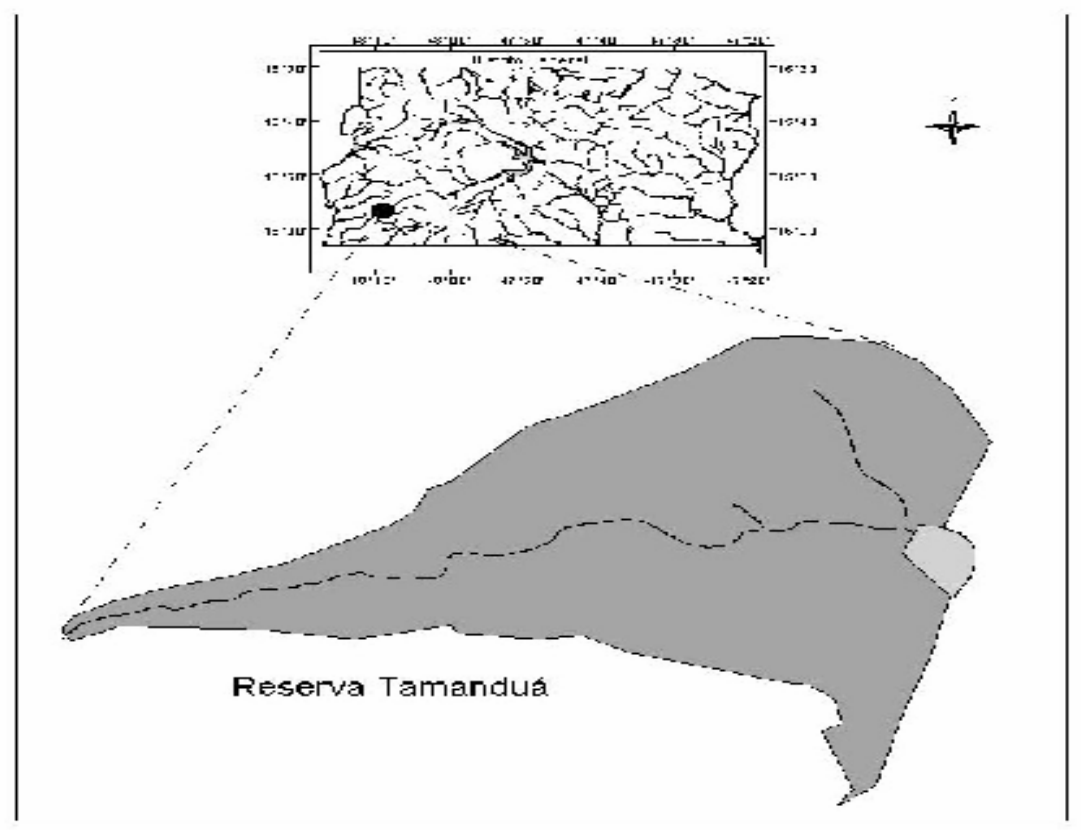

FIGURA 1: Posição geográfica da Reserva Genética Florestal Tamanduá no Distrito Federal.

FIGURE 1: Geographical location of the Tamanduá Forest Genetic Reserve at Federal District.

\section{Metodologia}

Os indivíduos arbóreos-arbustivos com circunferência à altura do peito (CAP) menor ou igual a 15 $\mathrm{cm}$ foram marcados, identificados e avaliados quanto à espécie botânica, altura total, CAP e circunferência de base $(\mathrm{CAB})$, quando esta se apresentasse maior ou igual a $5 \mathrm{~cm}$. Esses indivíduos, denominados de "jovens", foram considerados formadores do compartimento subbosque da Reserva, sendo mensurados e plotados em mapas, usando-se suas coordenadas cartesianas com a origem dos eixos ordenados no canto inferior esquerdo das parcelas de 20 x $10 \mathrm{~m}$ dos transectos (Silva et al., 2002).

Os indivíduos com $\mathrm{CAB}$ menor que $5 \mathrm{~cm}$, denominados de "regenerantes", foram identificados, enumerados e avaliados quanto aos parâmetros fitossociológicos e distribuição espacial. O estudo da distribuição espacial, entretanto, foi restringido às espécies consideradas prioritárias para conservação in situ.

Para avaliar a distribuição espacial dos regenerantes das espécies, foram utilizadas as medidas de dispersão de Morisita (I), sendo sua significância obtida pelo teste Qui-quadrado $\left(\chi^{2}\right)$, e a razão variância/média (R), de acordo com Brower e Zar (1977), Carvalho (1982) e Araújo et al. (2001).

Nesse caso, se $\mathrm{R}$ ou $\mathrm{I}=1$ diz-se que a distribuição da espécie é aleatória, se $\mathrm{R}$ ou $\mathrm{I}>1$, a distribuição apresenta padrão agregado e se $\mathrm{R}$ ou $\mathrm{I}<1$ a distribuição é uniforme.

A diversidade de espécies foi analisada pelo índice de Simpson, conforme Brower e Zar (1977).

A diversidade florística do componente indivíduos jovens foi expressa pelo índice de diversidade de Shannon (H'), conforme Brower e Zar (1977), Nappo (1999) e Mariano et al. (2000) e a composição florística dos regenerantes expressa pelo quociente de mistura de Jentsch, conforme Longhi (1980).

A análise estrutural do componente "regenerantes" e "jovens" foi efetuada pelos parâmetros 
fitossociológicos estimados pelo programa Fitopac 1, desenvolvido por Shepherd (1994).

\section{RESULTADOS E DISCUSSÃO}

\section{Florística e fitossociologia do componente regenerantes}

Foram encontrados 21.482 ind.ha $^{-1}$, identificadas 69 espécies vegetais, 53 gêneros e 39 famílias botânicas, conforme Silva et al., (2002).

Entre as famílias botânicas inventariadas, destacaram-se Leguminosae, contribuindo com dez espécies, Rubiaceae com oito espécies, Sapindaceae com sete e Meliaceae com seis espécies cada. Myrtaceae e Lauraceae, Annonaceae e Apocynaceae apareceram com cinco e quatro espécies, respectivamente. Essas famílias totalizaram cerca de 63,83\% das espécies amostradas na Reserva. (Silva et al., 2002).

Entre as espécies consideradas prioritárias para efeito deste trabalho (Tabela 1), representadas por regenerantes, destacaram-se Cariniana estrellensis com densidade absoluta de 1307 regenerantes ha ${ }^{-1} \mathrm{e}$ frequiência absoluta de 53,65, Apuleia leiocarpa com 400 regenerantes ha $^{-1}$ e frequêencia absoluta de 14,63\%. Copaifera langsdorffii com uma densidade absoluta de 321 regenerantes ha $^{-1} \mathrm{e}$ freqüência absoluta de 39,02, Anadenanthera macrocarpa com uma densidade absoluta de 273 regenerantes ha $^{-1}$ e frequiência absoluta de 31,70\%. Virola sebifera com 97 regenerantes e Hymenaea courbaril com 68 regenerantes ha ${ }^{-1}$ apareceram em seguida. Astronium fraxinifolium com 48 regenerantes e Schefflera morototoni com 29 regenerantes ha $^{-1}$ completaram a relação.

Esse resultado se assemelhou ao obtido no inventário dos indivíduos adultos efetuado nos transectos cuja ordem decrescente de dominância por família foi Leguminosae $(86,92 \%)$, Lecythidaceae $(9,94 \%)$, Myristicaceae $(1,76 \%)$, Araliaceae $(0,87 \%)$ e Anacardiaceae $(0,52 \%)$. As famílias Leguminosae e Lecythidaceae, neste caso, totalizaram 90,14\% do valor de importância (Silva et al., 2002).

TABELA 1: Parâmetros fitossociológicos dos regenerantes das principais espécies arbóreas na Reserva Genética Tamanduá, DF.

TABLE 1: Phytosociological parameters of regeneration of the main woody species at Tamanduá Forest Genetic Reserve, Brazil.

\begin{tabular}{ll|c|c|c|c}
\hline \multicolumn{1}{c|}{ Família } & \multicolumn{1}{c|}{ Nome científico } & DA & DR\% & FA & FR\% \\
\hline Lecythidaceae & Cariniana estrellensis & $1.307,32$ & 6,08 & 53,66 & 3,44 \\
Leg. Caesalpinioideae & Apuleia leiocarpa & 400,00 & 1,86 & 14,63 & 0,94 \\
Leg. Caesalpinioideae & Copaifera langsforffii & 321,95 & 1,50 & 39,02 & 2,50 \\
Leg. Mimosoideae & Anadenanthera macrocarpa & 273,17 & 10,27 & 31,71 & 2,03 \\
Myristicaceae & Virola sebifera & 97,56 & 0,45 & 21,95 & 1,41 \\
Leg. Caesalpinioideae & Hymenaea courbaril & 68,29 & 0,32 & 14,63 & 0,94 \\
Apocynaceae & Aspidosperma discolor & 58,54 & 0,27 & 12,19 & 0,78 \\
Anacardiaceae & Astronium fraxinifolium & 48,78 & 0,22 & 9,76 & 0,62 \\
Araliaceae & Schefflera morototoni & 29,27 & 0,14 & 7,32 & 0,47 \\
\hline
\end{tabular}

Em que: DA $=$ Densidade Absoluta; DR $=$ Densidade Relativa; $F A=$ Freqüência Absoluta; FR $=$ Frequiência Relativa.

\section{Distribuição espacial dos regenerantes}

Foram amostrados apenas dois indivíduos adultos de Schefflera morototoni e um de Astronium fraxinifolium, considerando-os raros na Reserva. Entretanto, deve-se salientar que uma espécie considerada rara em uma região pode ser comum em outra. Martins Netto (1992), nessa mesma área, constatou também a ausência de árvores adultas de Astronium fraxinifolium nas parcelas onde não ocorria regeneração natural da espécie. Schefflera morototoni, por ser uma espécie pioneira, apresentou também reduzida ocorrência no interior da mata, razão pela qual se constatou uma baixa frequiência da espécie na amostragem de adultos (Martins Netto, 1992). Outro aspecto a ser considerado por esse autor foi que após a dispersão de sementes, 
estas passaram a compor o banco de sementes e não o banco de plântulas, em face das condições inadequadas para germinação e posterior sobrevivência dessas duas espécies.

Para Auspurger (1983), Auspurger e Hogan (1983) e Guariguata e Pinard (1998), a distância máxima de dispersão de sementes depende do agente dispersor, quer seja ele vento, água ou animal. Assim, espécies cuja dispersão é anemocórica (Astronium fraxinifolium, Aspidosperma sp., Cariniana estrellensis) têm maior probabilidade de se regenerarem a consideráveis distâncias dos progenitores. Via de regra, as sementes/diásporos de espécies arbóreas tropicais se disseminam numa área irregular e restrita em torno da planta-mãe, com exceção das espécies com sementes aladas, diferentemente das que habitam regiões alagadas e daquelas dispersas por mamíferos, Auspurger e Hogan (1983)

Sementes dispersas pelo vento chegam a atingir distâncias maiores que $100 \mathrm{~m}$ da planta matriz em florestas não-perturbadas, mas a maioria (75\%) não alcança distância maior que $30 \mathrm{~m}$ da fonte (Guariguata e Pinard, 1998). Nesse caso, o conhecimento da direção dos ventos dominantes e da distribuição das clareiras são importantes para a organização de um planejamento para coleta de sementes, estudos da regeneração natural e recrutamento das espécies.

Semelhantemente, mais de $90 \%$ das sementes dispersas por animais se distribuem dentro de um raio de 30 m da planta matriz (Guariguata e Pinard, 1998), com exceção dos dispersores mamíferos como: morcegos, macacos e preguiças que conseguem levar o propágulo a distâncias maiores. De fato, Wehncke et al., (2003) examinaram o padrão de dispersão de sementes por macacos em Barro Colorado-Panamá e verificaram que a distância média de dispersão das sementes ingeridas era de $216 \mathrm{~m}$, havendo alta probabilidade de se localizarem entre 100 e 200 m distantes da planta-mãe.

Padrão de dispersão agregada, por exemplo, tem sido relacionado ao modo de dispersão de sementes, às condições edáficas e à estrutura da mata (Hubbell,1979; Ward e Parker, 1989).

Assim espécies, que possuem dispersão predominantemente barocórica, têm menos chance de se regenerarem longe dos progenitores. Isso foi observado para Hymenaea courbaril (dispersão mastocórica) que apareceu entre os regenerantes com uma densidade de sete regenerantes por hectare, em geral, situados próximos à planta-mãe, muito embora apresentasse, anualmente, grande produção de frutos. A maioria dos frutos, caindo próximo à planta-mãe, tem maior probabilidade de ser danificado por predadores habituais ou patógenos. Além disso, herbívoros de plântulas têm maior oportunidade de predar quando os indivíduos estão mais fortemente adensados. Todos esses fatores, aliados à competição natural por luz, nutrientes e água podem ter contribuído para que a densidade de Hymenaea courbaril fosse baixa. Não se encontrou, no inventário de jovens, nenhum indivíduo de Hymenaea courbaril, embora ocorresse com densidade de 13 árvores por hectare entre os adultos (Silva et al., 2002)

Nos padrões espaciais de distribuição dos regenerantes das espécies analisadas, constatou-se diferentes padrões de ocorrência (Tabela 2). Verificou-se que os indivíduos de Apuleia leiocarpa e Cariniana estrellensis apresentaram distribuição do tipo agregado, tanto pela medida (R) quanto pelo (I), confirmado pelo teste de qui-quadrado que rejeitou a hipótese de que a distribuição fosse aleatória. Esse padrão de distribuição de Apuleia leiocarpa e Anadenamthera macrocarpa se deve à dispersão barocórica de suas sementes. Aquino et al.(1999), trabalhando com Anadenamthera macrocarpa, em mata seca semidecídula de Minas Gerais, constataram que a chuva de sementes junto às plantas-mães e a alta de taxa de germinação foram os principais responsáveis pelo padrão de distribuição agrupada. Virola sebifera, de dispersão ornitocórica, apresentou distribuição do tipo regular-uniforme pelos dois índices analisados, resultado esse também encontrado na mesma Reserva por Martins Netto (1992). Para Schefflera morototoni não foi possível calcular os índices de dispersão, em razão do pequeno número de indivíduos amostrados, porém, Martins Netto (1992) encontrou tendência à agregação para a espécie na Reserva Genética Florestal Tamanduá.

Astronium fraxinifolium e Aspidosperma discolor apresentaram distribuição uniforme pela medida (R) e distribuição agregada pelo Índice de Morisita, confirmadas também pelo teste de qui-quadrado. Considerando-se que a dispersão de suas sementes é tipicamente anemocórica, essas espécies têm maior chance de se regeneraram longe das matrizes, conforme evidenciado por Martins Netto (1992). Entretanto, os 
regenerantes emergindo em regiões mais distantes da planta-mãe poderão encontrar ambientes menos protegidos e, assim, apresentarem taxa de mortalidade inicial relativamente alta e, conseqüientemente, apresentarem distribuição menos agregada. A medida de dispersão (R) parece refletir melhor essa tendência, nesse caso. Aqueles indivíduos, que conseguirem se estabelecer efetivamente, terão probabilidade maior de sobrevivência, seja pela baixa densidade coespecífica, o que reduziria a competição, seja pela baixa frequiência de predadores específicos (Martins Netto,1992).

Copaifera langsdorffii e Hymenaea courbaril apresentaram comportamentos semelhantes pelos dois índices analisados, ou seja, dispersão uniforme pela medida (R) e agregada pelo (I). Considerando-se o tipo de dispersão de suas sementes (barocórica/mastocórica), a agregação parece ser mais adequada a essas espécies que têm menos chances de se regenerarem longe dos progenitores. Walter et al (1997) constataram, na mata ciliar da Fazenda Sucupira, DF, que a maioria dos regenerantes de Copaifera langsdorffii se encontrava sob a planta-referência.

TABELA 2: Padrão de distribuição espacial dos regenerantes das espécies-alvo na Reserva Genética Florestal Tamanduá, DF, em função da razão/variância (R) e do índice de Morisita (I).

TABLE 2: Spatial distribution pattern of regenerants of the target species at Tamanduá Forest Genetic Reserve, Brazil, as a function of ratio/variance (R) and Morisita index (I) .

\begin{tabular}{l|c|c|c}
\hline Nome Científico & $\mathrm{R}$ & $\mathrm{I}$ & \multicolumn{1}{c}{$\chi^{2}$} \\
\hline Anadenanthera macrocarpa & $2,90 \mathrm{~A}$ & $5,86 \mathrm{~A}$ & $171,1^{*}$ \\
Copaifera langsdorffii & $0,32 \mathrm{U}$ & $1,63 \mathrm{~A}$ & $60,2^{*}$ \\
Apuleia leiocarpa & $10,43 \mathrm{~A}$ & $14,85 \mathrm{~A}$ & $594,0^{*}$ \\
Astronium fraxinifolium & $0,20 \mathrm{U}$ & $4,10 \mathrm{~A}$ & $52,4^{*}$ \\
Cariniana estrellensis & $12,30 \mathrm{~A}$ & $1,94 \mathrm{~A}$ & $758,9^{*}$ \\
Hymenaea courbaril & $0,14 \mathrm{U}$ & $1,95 \mathrm{~A}$ & $45,7^{*}$ \\
Aspidosperma discolor & $0,17 \mathrm{U}$ & $2,73 \mathrm{~A}$ & $48,6^{*}$ \\
Virola sebifera & $0,10 \mathrm{U}$ & $0,91 \mathrm{U}$ & $39,2^{*}$ \\
\hline
\end{tabular}

Em que: * Significativo ao nível de $1 \% ; \mathrm{A}=$ Agregado; $\mathrm{U}=$ Uniforme; $\chi^{2}=$ qui quadrado.

\section{Florística e fitossociologia dos jovens}

Foram inventariados 2055 inds. ha $^{-1}$ na Reserva referentes a 33 espécies e 21 famílias botânicas (Tabela 3). Constatou-se que apenas Copaifera langsdorffii e Virola sebifera, relacionadas como regenerantes, ocorreram no levantamento de jovens, apresentando valor de importância de 6,22\% e 2,90\% respectivamente. Espécies emergentes como Hymenaea courbaril e Anadenanthera macrocarpa não apresentaram indivíduos nas menores classes de diâmetro (Tabela 3). Cariniana estrellensis, considerada tolerante, apesar de apresentar alta densidade de regenerantes e ocupar o dossel da floresta, não foi encontrada entre os jovens. Não obstante a ocorrência de árvores matrizes das espécies Hymenaea courbaril e Anadenanthera macrocarpa na Reserva, com regular produção anual de sementes e considerável produção de plântulas, não se constataram suas presenças como indivíduos jovens. Apuleia leiocarpa, que tem seu estabelecimento facilitado pela abertura de clareiras naturais e/ou antropizadas, também não foi encontrada entre os jovens. Fato semelhante ocorreu com Anadenanthera macrocarpa e Hymenaea courbaril, apesar da grande ocorrência de regenerantes observada no levantamento.

As famílias mais importantes na composição do sub-bosque, constituído por indivíduos jovens (Tabela 4), foram, na ordem decrescente de dominância relativa, Meliaceae $(33,06 \%)$, Burseraceae $(15,02 \%)$, Rubiaceae $(14,83 \%)$,Rutaceae $(6,78 \%)$ e Hippocrateaceae $(5,61 \%)$. Trichilia elegans foi a espécie mais abundante do sub-bosque, seguida de Tetragastris sp. e Trichilia claussenii ( Figura 2), confirmando o caráter marcante da família Meliaceae na constituição do sub-bosque. 


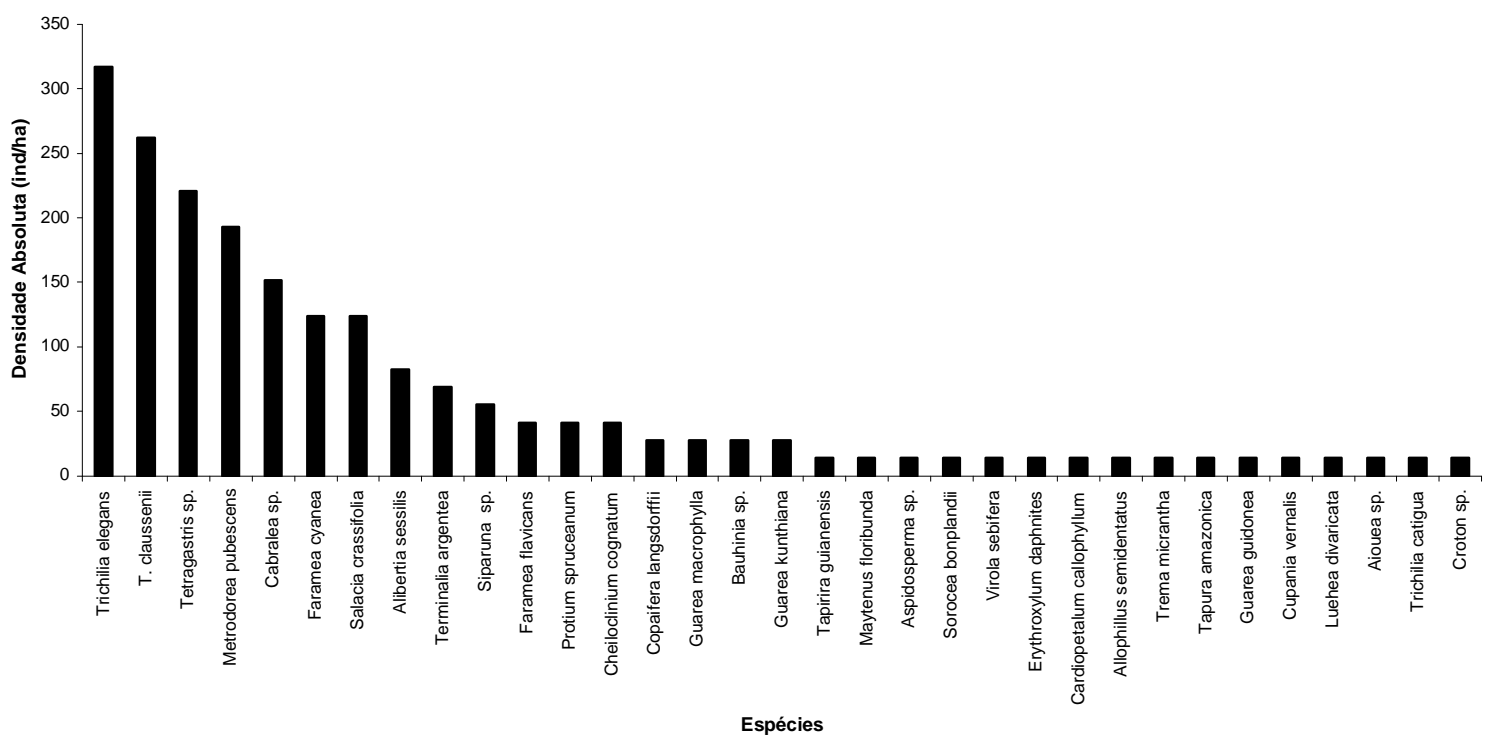

FIGURA 2: Densidade populacional de jovens na Reserva Genética Florestal Tamanduá, DF.

FIGURE 2: Young individual species population at Tamandua Forest Genetic Reserve, Brazil.

TABELA 3: Parâmetros fitossociológicos das espécies de jovens amostradas com $15 \mathrm{~cm}<\mathrm{CAP}>5 \mathrm{~cm}$, na Reserva Genética Florestal Tamanduá, DF.

TABLE 3: Phytosociological parameters of young individual species with $15 \mathrm{~cm}<\mathrm{gbh}>5 \mathrm{~cm}$ at Tamandua Forest Genetic Reserve, Brazil.

\begin{tabular}{l|c|c|c|c|c}
\hline \multicolumn{1}{c}{ Nome científico } & DA & DR & DoR & FR & VI \\
\hline Trichilia elegans & 317,2 & 15,44 & 12,98 & 12,79 & 41,21 \\
Tetragastris sp. & 220,7 & 10,74 & 12,11 & 9,30 & 32,16 \\
Trichilia claussenii & 262,1 & 12,75 & 11,95 & 5,81 & 30,52 \\
Metrodorea pubescens & 193,1 & 9,4 & 6,78 & 8,14 & 24,31 \\
Cabralea sp. & 151,7 & 7,38 & 5,45 & 9,30 & 22,14 \\
Faramea cyanea & 124,1 & 6,04 & 5,23 & 6,98 & 18,24 \\
Alibertia sessilis & 82,8 & 4,03 & 6,24 & 4,65 & 14,92 \\
Salacia eliptica & 124,1 & 6,04 & 4,3 & 1,16 & 11,51 \\
Terminalia argentea & 69 & 3,36 & 4,62 & 3,49 & 11,46 \\
Siparuna sp. & 55,2 & 2,68 & 5,58 & 2,33 & 10,59 \\
Faramea flavicans & 41,4 & 2,01 & 3,35 & 3,49 & 8,86 \\
Protium spruceanum & 41,4 & 2,01 & 2,91 & 2,33 & 7,25 \\
Cheiloclinium cognatum & 41,4 & 2,01 & 1,31 & 3,49 & 6,81 \\
Copaifera langsdorffii & 27,6 & 1,34 & 2,55 & 2,33 & 6,22 \\
Guarea macrophylla & 27,6 & 1,34 & 0,57 & 2,33 & 4,24 \\
Bauhinia sp. & 27,6 & 1,34 & 0,53 & 2,33 & 4,28 \\
Guarea kunthiana & 27,6 & 1,34 & 1,29 & 1,16 & 3,79 \\
Tapirira guianensis & 13,8 & 0,67 & 1,86 & 1,16 & 3,70 \\
Maytenus floribunda & 13,8 & 0,67 & 1,54 & 1,16 & 3,37 \\
Aspidosperma sp. & 13,8 & 0,67 & 1,32 & 1,16 & 3,15 \\
Sorocea bonplandii & 13,8 & 0,67 & 1,29 & 1,16 & 3,13 \\
Virola sebifera & 13,8 & 0,67 & 1,07 & 1,16 & 2,90 \\
Erythroxylum daphnites & 13,8 & 0,67 & 0,69 & 1,16 & 2,52 \\
\hline
\end{tabular}


TABELA 3: Continuação ...

TABLE 3: Continued ...

\begin{tabular}{l|c|c|c|c|c}
\hline \multicolumn{1}{c}{ Nome cientifico } & DA & DR & DoR & FR & VI \\
\hline Cardiopetalum callophyllum & 13,8 & 0,67 & 0,6 & 1,16 & 2,44 \\
Allophillus cemidentatus & 13,8 & 0,67 & 0,55 & 1,16 & 2,39 \\
Trema micrantha & 13,8 & 0,67 & 0,52 & 1,16 & 2,36 \\
Tapura amazonica & 13,8 & 0,67 & 0,52 & 1,16 & 2,36 \\
Guarea guidonea & 13,8 & 0,67 & 0,43 & 1,16 & 2,26 \\
Cupania vernalis & 13,8 & 0,67 & 0,41 & 1,16 & 2,24 \\
Luehea divaricata & 13,8 & 0,67 & 0,39 & 1,16 & 2,22 \\
Aiouea sp. & 13,8 & 0,67 & 0,39 & 1,16 & 2,22 \\
Trichilia catigua & 13,8 & 0,67 & 0,39 & 1,16 & 2,22 \\
Croton sp. & 13,8 & 0,67 & 0,27 & 1,16 & 2,10 \\
Total & 2055,17 & 100,00 & 100,00 & 100,00 & - \\
\hline
\end{tabular}

Em que: DA = Densidade Absoluta; DR $=$ Densidade Relativa; DoR = Dominância Relativa; FR = Freqüência Relativa; VI = Valor de Importância.

TABELA 4: Parâmetros fitossociológicos das famílias de jovens amostradas na Reserva Genética Florestal Tamanduá.

TABLE 4: Phytosociological parameters of young families of individuals in the Tamanduá Forest Genetic Reserve, Brazil

\begin{tabular}{lcc|c|c}
\hline Família & DR & DoR & FR & VI \\
\hline Meliaceae & 39,54 & 33,06 & 25,68 & 98,33 \\
Rubiaceae & 12,08 & 14,83 & 14,86 & 41,77 \\
Burseraceae & 12,76 & 15,02 & 13,51 & 41,29 \\
Rutaceae & 9,39 & 6,78 & 9,46 & 25,63 \\
Hippocrateaceae & 8,05 & 5,61 & 5,41 & 19,07 \\
Combretaceae & 3,36 & 4,62 & 4,05 & 12,03 \\
Leguminosae & 3,68 & 3,09 & 5,41 & 11,18 \\
Monimiaceae & 2,69 & 5,58 & 2,7 & 10,97 \\
Sapindaceae & 1,35 & 0,96 & 2,7 & 5,01 \\
Anacardiaceae & 0,67 & 1,86 & 1,35 & 3,88 \\
Celastraceae & 0,67 & 1,54 & 1,35 & 3,56 \\
Apocynaceae & 0,67 & 1,32 & 1,35 & 3,34 \\
Moraceae & 0,67 & 1,29 & 1,35 & 3,32 \\
Myristicaceae & 0,67 & 1,07 & 1,35 & 3,09 \\
Annonaceae & 0,67 & 0,60 & 1,35 & 2,63 \\
Dichapetalaceae & 0,67 & 0,52 & 1,35 & 2,55 \\
Ulmaceae & 0,67 & 0,52 & 1,35 & 2,55 \\
Lauraceae & 0,67 & 0,39 & 1,35 & 2,41 \\
Tiliaceae & 0,67 & 0,39 & 1,35 & 2,41 \\
Euphorbiaceae & 0,67 & 0,27 & 1,35 & 2,29 \\
\hline
\end{tabular}

Em que: DoR = Dominância Relativa; FR = Frequiência Relativa; VI = Valor de Importância; DR=Densidade Relativa.

A estrutura populacional do componente indivíduos jovens indicou forma decrescente do tipo $\mathrm{J}$ invertido ( Figura 3), em que se verificou a predominância de seus indivíduos na primeira classe de CAP (5$6 \mathrm{~cm}$ ). A distribuição de diâmetros e o processo de regeneração natural constituem características importantes do estoque de plantas do componente jovem de uma floresta. Martins Netto (1992) analisou aspectos demográficos de Astronium fraxinifolium, Schefflera morototoni e Virola sebifera nessa Reserva e concluiu que, apesar dessas espécies apresentarem populações com distribuição de diâmetro de base na forma de $\mathbf{J}$ invertido, o número reduzido de indivíduos nas classes acima de quatro centímetros poderia significar que o ciclo de vida não estaria se completando, ou que tais espécies estariam em latência no banco de plântulas. Os 
dados apresentados na Figura 3 sugerem que o desenvolvimento dos indivíduos das espécies arbóreasarbustivas, formadoras do sub-bosque da Reserva, estava ocorrendo com tendência à forma regular. Entretanto, para garantir a sobrevivência das espécies analisadas seria interessante efetuar algum tipo de intervenção silvicultural, como por exemplo, eliminação de cipós, a fim de favorecer o seu estabelecimento no sub-bosque, uma vez que se encontravam apenas presentes entre os regenerantes inventariados.

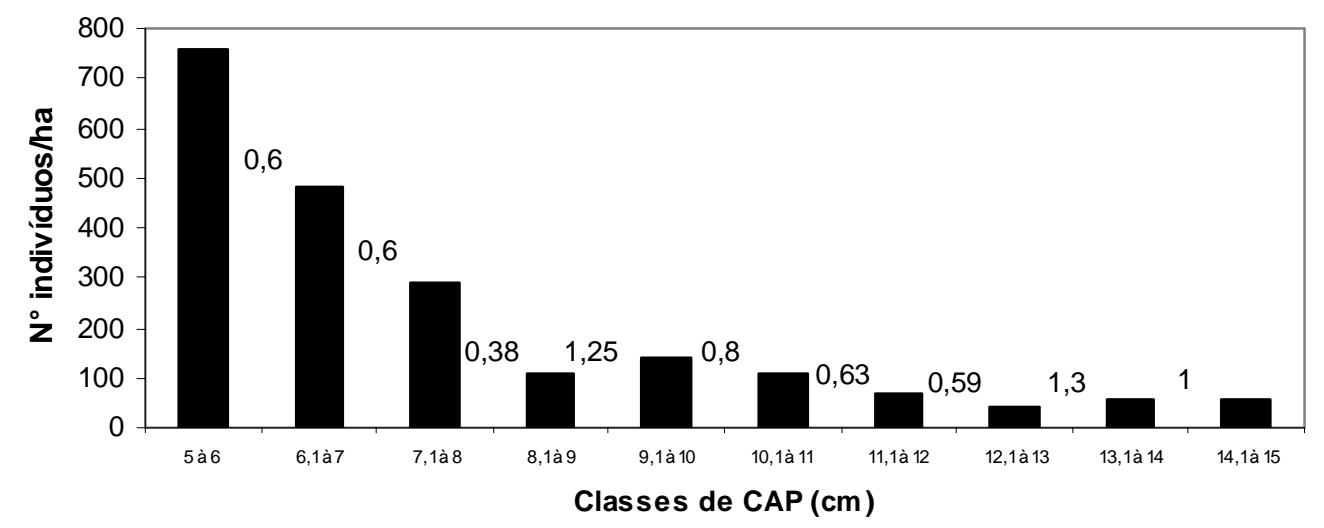

FIGURA 3: Distribuição de indivíduos jovens por classe de circunferência na Reserva Genética Florestal Tamanduá no ano 2000.

FIGURA 3: Distribution of young individuals by girth classes at Tamandua Forest Genetic Reserve, Brazil.

A altura média dos indivíduos jovens amostrados foi de $3,53 \mathrm{~m} \pm 1,53 \mathrm{~m}$, indicando condições de sombreamento relativamente denso, tendo-se em conta a densidade de indivíduos ocorrentes na área. Trichilia elegans e Trichilia clausenii, apesar de serem espécies mais abundantes no sub-bosque, apresentaram alturas médias inferiores à média geral. Alibertia sessilis foi a espécie com maior altura média observada. Considerando-se as espécies, individualmente, constatou-se que Trichilia clausenii, Alibertia sessilis e Tapirira guianensis apresentaram maiores valores de altura cuja variação oscilou entre 7,0 a $8,0 \mathrm{~m}$. Copaifera langsdorffii e Virola sebifera apresentaram também alturas individuais superiores à média, porém com baixo valor de importância. A Figura 4 mostra a amplitude de variação das alturas observadas para um grupo selecionado de espécies com indivíduos jovens inventariados na Reserva, utilizando-se o programa STATISTICA (1995).

O índice de Shannon calculado foi de $\mathrm{H}^{\prime}=2,89$ nats. ind. ${ }^{-1}\left(\mathrm{~J}^{\prime}=82,6 \%\right)$ para os jovens, semelhante ao encontrado por Nascimento et al. (2000) na região central do Rio Grande do Sul ( $\mathrm{H}^{\prime}=2,90$ nats. ind. $\left.{ }^{-1}\right)$, apesar das diferenças existentes entre as condições edafo-climáticas das regiões, indicando que a Reserva em questão contém considerável diversidade florística. Esse resultado está também bastante próximo do valor de 2,51 nats. ind. ${ }^{-1}$ encontrado por Silva Júnior et al. (1998) e de 2,84 nats. ind. ${ }^{-1}$ encontrado por Guarino et al. (2001) para trechos de matas de galeria no Brasil Central. Os valores do índice de diversidade de Shannon usualmente ficam entre 1,5 e 3,5 e, raramente, ultrapassam 4,5, conforme Dias et al. (2000). O índice de Simpson foi de 0,927 , indicando tratar-se de baixa dominância de espécies entre os jovens.

Os valores obtidos para o Quociente de Mistura indicaram uma relação média de 1:3; o que significa que há uma grande heterogeneidade florística na regeneração natural na Reserva. Finol (1975), citado por Longhi (1980), encontrou, para as florestas tropicais venezuelanas, uma proporção de 1:9. Pode-se inferir, portanto, que a Reserva Genética Tamanduá é considerada mais rica em espécies que aquelas analisadas pelos autores mencionados. 

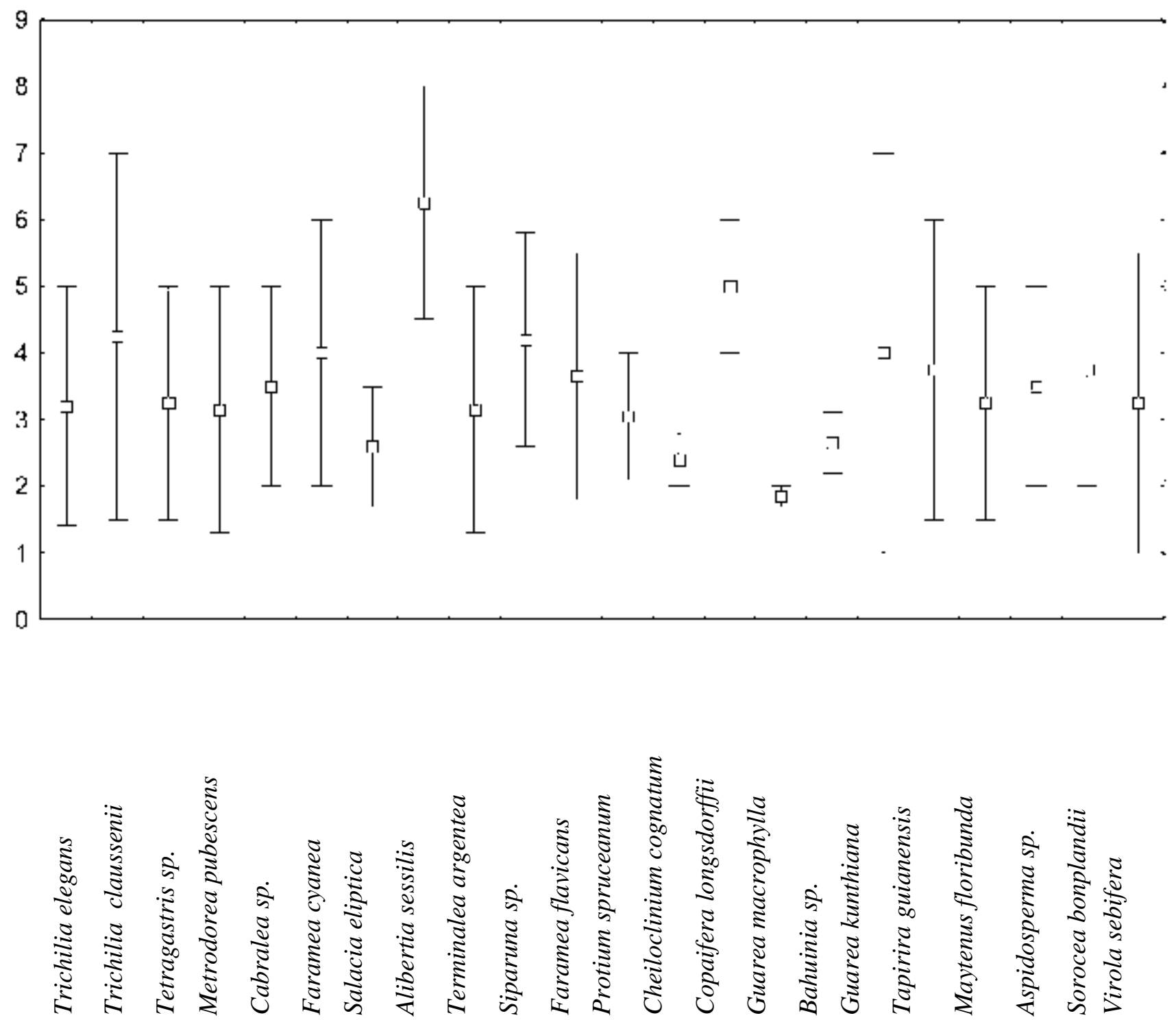

FIGURA 4: Amplitude de variação de alturas dos indivíduos jovens da Reserva Genética Florestal Tamanduá, DF.

FIGURE 4: Range of height variation of young individuals measured at Tamandua Forest Genetic Reserve, Brazil.

\section{CONCLUSÕES}

Conforme os resultados, pôde-se constatar que todas as espécies, objeto deste trabalho, ocorreram entre os regenerantes, constituindo, de certa forma, um indicativo para sua permanência na floresta. Constatou-se, entretanto, que apenas Copaifera langsdorffii e Virola sebifera relacionadas como regenerantes ocorreram entre os indivíduos jovens, mesmo assim, com baixo valor de importância.

Os indivíduos jovens das diversas espécies arbóreo-arbustivas inventariadas, formadores do subbosque da Reserva, mostraram forma de distribuição de diâmetro decrescente do tipo $\mathrm{J}$ invertido, com tendência à forma regular, o que poderia indicar estabilidade e adaptação ao ambiente. $\mathrm{O}$ desenvolvimento e adaptação dessas espécies estariam criando condições propícias para o estabelecimento futuro de espécies mais tolerantes e, possivelmente, mais importantes sob o ponto de vista silvicultural.

A Reserva Genética Florestal Tamanduá pode ser considerada rica em espécies, uma vez que o quociente de mistura foi de 1:3, o que significa que há uma grande heterogeneidade florística na Reserva. 
Meliaceae foi a família mais abundante amostrada entre os jovens, sendo Trichilia elegans, a espécie com maior participação ( $\mathrm{DoR}=12,98 \%$ ). A família Leguminosae foi, contudo, a mais importante entre os regenerantes na formação do sub-bosque, contribuindo com Anadenanthera macrocarpa, Apuleia leiocarpa, Copaifera langsdorffii e Hymenaea courbaril.

\section{REFERÊNCIAS BIBLIOGRÁFICAS}

AQUINO, F. de G.; OLIVEIRA, M.C. de; SCHIAVINI, I.; RIBEIRO, J.F. Dinâmica de população de Anadenanthera macrocarpa e Acacia glomerosa em mata seca semi-decídua na Estação Ecológica do Panga. (Uberlândia-MG). Boletim do Herbário Ezechias Paulo Heringer, Brasília v. 4, p. 90-102, 1999.

ARAÚJO, M.M.; OSAQUI, H; MELO, R.S.de. Padrão de distribuição espacial de castanheira (Bertholettia excelsa H.B.K), Barragem do Gelado, Floresta Nacional de Carajás - Pará. In: SIMPÓSIO LATINO AMERICANO SOBRE MANEJO FLORESTAL, 2., 2001, Santa Maria. Anais... Santa Maria: Universidade Federal de Santa Maria. 2001. p. 367-375.

AUGSPURGER, C.K. Seed dispersal of the tropical tree, Platypodium elegans, and the escape of its seedings from fungal pathogens. Journal of Ecology,. v.71, p.759-771. 1983.

AUGSPURGER, C.K..; HOGAN, K. P. Wind dispersal of fruits with variable seed number in a tropical tree (Lonchocarpus pentaphyllus: Leguminosae). American Journal of Botany, v.70, p.1031-1037, 1983.

AYOADE, J. O. Introduction to climatology for the tropics. Clichester: J. Wiley \& Son; 1983. 258p.

BROWER, J.E.; ZAR, J.H. Field and laboratory methods for general ecology. Iowa: Wm. C. Brown Co., 1977. $194 \mathrm{p}$.

CARVALHO, J.O.P. Análise estrutural da regeneração natural em floresta tropical densa na região de Tapajós no Estado do Pará. 1982 . 63p. Dissertação (Mestrado)- Universidade Federal do Paraná, Curitiba, 1982.

DIAS, A.C.; CUSTÓDIO FILHO, A.; FRANCO, G.A.D.C. Diversidade do componente arbóreo, em floresta pluvial Atlântica secundária, São Paulo, Brasil. Revista do Instituto Florestal de São Paulo, v. 12, n. 2, p.127-153, 2000.

DIAS, B.F. de S. Fundação Pró-Natureza: alternativas de desenvolvimento dos cerrados: manejo e conservação dos recursos naturais renováveis. Brasília: IBAMA, 1992. 97p.

EITEN, G. Vegetação do Cerrado._In: PINTO, M. N. (Org.). Cerrado: caracterização, ocupação e perspectivas. Brasília: Universidade de Brasília, 1990. p. 9-65.

FONSECA, G.A.B. Fauna nativa. In: DIAS, B.F. de S. Fundação Pró-Natureza: alternativas de desenvolvimento dos cerrados: manejo e conservação dos recursos naturais renováveis. Brasília: IBAMA. 1992. 97p.

GUARIGUATA, M.R. ; PINARD. M. A. Ecological knowledge of regeneration from seed in neotropical Forest trees: Implication for natural Forest management. Forest Ecology Management, v.112, p. 87-99, 1998.

GUARINO, E.S.G.; SALES, P.A.; PEREIRA, J.B.; WALTER, B.M.T. Comparação de dois trechos de Matas de Galeria no Distrito Federal, Brasil, (Comparison between two flooded stretch Gallery Forest in the Federal District, Brazil). In: ENCONTRO DO TALENTO ESTUDANTIL, 6., Brasilia, 2001. [Anais...] Brasília: Embrapa Recursos Genéticos e Biotecnologia, 2001. p.64.

HUBBELL, S.P. Tree special, abundance diversity in a tropical dry forest. Science, v. 203, n. 4387, p.1299-1309, 1979.

KAGEYAMA, P. Y. ; PATIÑO-VALERA, F. Conservación y manejo de recursos forestales: factores que influyen em la estructura y diversidad de los ecosistemas forestales. México,1985. 24p. Documento apresentado ao IX Congresso Florestal Mundial.

LONGHI, S.J. A estrutura de uma floresta natural de Araucaria angustifolia (Bert.) O. Ktze no sul do Brasil., 1980. 198 p. Dissertação (Mestrado) - Universidade do Paraná, Curitiba, 1980.

MARIANO, G; CRESTANA, C. de S.M.; GIANNOTTI, E.; BATISTA, E.A. Fitossociologia da regeneração natural sob plantio heterogêneo em Piracicaba - SP. Revista do Instituto Florestal de São Paulo, v.12, n. 2, p. 167-177, 2000.

MARTINS NETTO, D.A. Aspectos demográficos de quatro espécies florestais na mata galeria da Reserva de Genética Tamanduá-DF. 1992. 96p. Dissertação (Mestrado)- Universidade de Brasília, Brasília, 1992. 
MORY, A. de M. ; JARDIM, F. C. da S. Comportamento de Goupia glabra Aubl. (Cupiúba) em diferentes níveis de desbastes por anelamento em plantas naturais. Revista de Ciências Agrárias, Belém, n. 36, p. 55-66, 2001.

NAPPO, M.E. Inventário florístico e estrutural da regeneração natural no sub-bosque de povoamentos homogêneos de Mimosa scabrella Benthan, implantados em áreas mineradas em Poços de Caldas, MG. 1999. 87p. Dissertação (Mestrado)- Universidade Federal de Lavras, Lavras, 1999.

NASCIMENTO, A.R.T.; LONGHI, S.J., ALVAREZ FILHO, A. ; GOMES, G.S. Análise da diversidade florística e dos sistemas de dispersão de sementes em um fragmento florestal na região central do Estado do Rio Grande do Sul. NAPAEA, n. 12, p. 49-67, 2000.

PAULA, J.E. de; ENCINAS, J.I; MENDONÇA, R.C. de; LEÇO, D.T. Estudo dendrométrico e ecológico de mata ripária da região Centro-Oeste. Pesquisa Agropecuária Brasileira, v. 25, n. 1, p. 43-55, 1990.

ROCHE,. L. Forest genetic conservation. Brasil/Bangor: IICA, 1987. 37p. (IICA; EMBRAPA. Report on the threeweek consultancy).

SHEPHERD, G. J. Fitopac 1: manual do usuário. Campinas: UNICAMP, Departamento de Botânica, 1994. 9p.

SCHIAVINI, I. Estrutura das comunidades arbóreas de mata de galeria da Estação Ecológica do Panga (Uberlândia-MG). 1992. 133p. Tese (Doutorado) - Universidade de Campinas, Campinas, 1992.

SILVA, J. A. da; LEITE, E. J.; ARMANDO, M. S.; NORONHA, S. E. de; REZENDE, J. M. Monitoramento da Reserva Genética Florestal Tamanduá. Brasília: Embrapa Recursos Genéticos e Biotecnologia, 2002. 40p. (Documentos, 86).

SILVA JÚNIOR, M.C.; FELFILI, J.M.; SILVA, P.E.N.; RESENDE, A.V. Análise florística de matas de galeria no Distrito Federal. In: RIBEIRO, J.F. (Ed.) Cerrado: matas de galeria. Planaltina: EMBRAPA-CPAC, 1998. p. 52-84.

STATSOFT, INC. Statistica for windows: computer program manual. Tulsa OK, 1995.

WARD, J.S. ; PARKER, G.R. Spatial dispersion of woody regeneration in an old-growth forest. Ecology, v. 70, n. 5, p. $1279-1285,1989$.

WALTER, B.M.T; PINHO, G.S.C. de; SAMPAIO, A.B.; CIAMPI, A. Y. Estrutura populacional de Copaifera langsdorffii na mata do Açudinho, Fazenda Sucupira - DF. Brasília: Embrapa Recursos Genéticos e Biotecnologia, 1997. 8p. (Comunicado Tecnico, 22)

WEHNCKE, E. V.; HUBELL, S. P.; FOSTER, R. B.; DALLING, J. W. Seed dispersal patterns proudced by whitefaced monkeys: implications for the dispersal limitation of neotropical trees species. Journal of Ecology, v. 91, n. 4, p. $677,2003$.

WHITMORE, T. C. A review of some aspects of tropical rain forest seedling ecology with suggestions for further enquiry. In: Swainer, M. D. The ecology of tropical rain forest tree seedling. Paris: UNESCO, 1996. p. 3-39. 